\section{Self-Consistency}

On the one hand we have to study the connection which the hydrodynamical equations of motion establish between gravitation field and statistical distribution function $f$. On the other hand we have to satisfy Poisson's equation for the gravitation potential and thereby secure a self-consistent field. This problem is, however, so complicated that the usual hydrodynamical methods have to stop half-way, ignoring the Poisson equation and therefore self-consistency. The main idea of the transformation theory developed in Part II is to provide a mathematical shortcut which makes it possible to attack dynamically more complicated problems, and also self-consistent fields; an integration of the wave

ticular sample of clusters as we encounter them today, but not to a statistical distribution of clusters in the big system. The latter is represented by a "super" $\psi$ wave which tells nothing about internal structure of the clusters. equation is attainable even for rather complicated gravitation fields. With this powerful mathematical tool we may hope not only to calculate self-consistent steady fields but selfconsistent transient fields. In particular we are interested in the explanation of the asymptotic behavior of stellar systems like the flattening process of a galaxy.-Our solar system seems to have developed from a more chaotic distribution of masses, which first became flattened into a disk shaped body and which through their interactions had a tendency to group into preferential orbits. To such a system it is possible to apply a wave-mechanical hydrodynamical description. There is a vast field of dynamically more complicated problems about our solar system (restricted problems of 4 bodies, resonance problems, and others) which in this way become accessible to solution merely because of the simplicity of our mathematical tool.

PHYSICAL REVIEW VOLUME 70, NUMBERS 7 AND 8 OCTOBER 1 AND 15,1946

\title{
Properties of 94(239)
}

J. W. Kennedy, G. T. Seaborg, E. Segrè, and A. C. Wahl Radiation Laboratory and Department of Chemistry, University of California, Berkeley, California

(Received May 29, 1941)*

$\mathrm{W}^{\mathrm{E}}$ E would like to report that we have observed the fission of $94^{239}$ with slow neutrons. The cross secton for the fission of $94^{239}$ with slow neutrons is even larger than that of $\mathrm{U}^{235}$. The cross section was determined by comparing the number of fissions obtained with a sample containing $94^{239}$ with the number obtained with a sample of ordinary uranium under conditions which were as identical as possible in every detail. Two separate methods were used to prove that the fissions in the $94^{239}$ sample could not be attributed to the presence of uranium impurity: (1) complete and identical chemical tests on the isolation of rare earth carrier material for element 94 from non-irradiated uranium, i.e., "blank tests," in which the absence of uranium impurity in the final product was established by showing

* This letter was received for publication on the date indicated but was voluntarily withheld from publication until the end of the war. The original text has been somewhat changed, by omissions, in order to conform to present declassification standards. the absence of uranium alpha-particles and (2) demonstration that the ratio of the cross section for slow neutrons to that for fast neutrons for the fission of $94^{239}$ is different from the same ratio for natural uranium. This latter experiment has not yet been carried out in its optimum form, and we hope to submit a later report giving details of an improved experiment.

The details follow:

A sample of uranyl nitrate $\left(\mathrm{UO}_{2}\left(\mathrm{NO}_{3}\right)_{2} \cdot 6 \mathrm{H}_{2} \mathrm{O}\right)$ weighing 1.2 kilograms was distributed in a large paraffin block, placed directly behind the beryllium target of the 60-inch Berkeley cyclotron, and given over a period of about two days a 3500 microampere-hour bombardment with neutrons from beryllium plus $16-\mathrm{Mev}$ deuterons. This uranyl nitrate was placed in a continuously operating glass extraction apparatus, two liters of diethyl ether were added, and practically all of the uranyl nitrate was extracted into the ether phase. The $93^{239}$ was isolated from the aqueous 
phase with rare earth fluoride carrier by the method of McMillan and Abelson ${ }^{1}$ and the mixture of $3 \mathrm{mg}$ of $\mathrm{La}$ and $3 \mathrm{mg}$ of $\mathrm{Ce}$ which was "carrying" the $93^{239}$ was reprecipitated as fluoride six times in order to remove any uranium impurity. This sample of $93^{239}$ at the time of its purification (which included purification from element 94) had an activity of 125 millicuries as determined with the aid of an ionization chamber, connected to an FP-54 vacuum tube electrometer, which had been calibrated in an absolute manner for $93^{239}$ radiation by the use of a Geiger counter and the method of aliquots. After the $93^{239}$ had decayed into $94^{239}$, preliminary fission tests were made on this sample which then contained 0.5 microgram of $94^{239}$. This sample was placed near the screen window of an ionization chamber which was imbedded in paraffin near the beryllium target of the 37-inch Berkeley cyclotron. This gave a small, but detectable, fission rate when a 6-microampere beam of deuterons was used. However, this count was too small to be sure that the fissions were not caused by uranium impurity and the fission rate with fast neutrons (i.e., when the chamber was surrounded with cadmium) was too small to determine with any accuracy at all.

This sample, which had a thickness of total material amounting to $4.8 \mathrm{mg}$ per $\mathrm{cm}^{2}$, was then subjected to a chemical procedure ${ }^{2}$ designed to isolate the $94^{239}$ in much less material. The procedure was carefully tested in blank experiments with the aid of element 94 from deuteronactivated uranium as tracer to show that the yield was practically 100 percent. In this manner the 0.5 microgram of $94^{239}$ was isolated with lanthanum fluoride in which the thickness of total material amounted to $0.16 \mathrm{mg}$ per $\mathrm{cm}^{2}$. (In a blank experiment in which the same amount of lanthanum fluoride was isolated from non-irradiated uranium by an identical chemical procedure the alpha-count was less than $\frac{1}{4}$ per minute. Cor-

\footnotetext{
${ }^{1}$ E. M. McMillan and P. H. Abelson, Phys. Rev. 57, $1185(1940)$

${ }^{2}$ G. T. Seaborg, A. C. Wahl, and J. W. Kennedy, Phys. Rev. 69, 367 (1946).
}

recting for the geometrical factor, as determined with the aid of a known amount of uranium of the same thickness, this means that the sample contained less than 3 micrograms of $U^{238}$ impurity and hence less than 0.03 microgram of $\mathrm{U}^{235} \mathrm{im}$ purity. This is the upper limit of uranium impurity which may be present, as determined by the limitations on the method of detection, and the actual amount might very well be much less than this.) A standard uranium sample, probably in the form of anhydrous oxide, of a thickness and area very nearly the same as that of the $94^{239}$ sample, was prepared by the electolysis of ordinary uranium out of absolute ethyl alcohol solution. This uranium standard contained $200 \mathrm{mi}$ crograms of $\mathrm{U}^{238}$ and hence 1.46 micrograms of $\mathrm{U}^{235}$. The easily measurable fission rates of the 0.5 microgram $94^{239}$ sample and the 1.46 microgram $\mathrm{U}^{235}$ sample were compared when placed near the screen window of an ionization chamber imbedded in paraffin near the beryllium target of the 37-inch Berkeley cyclotron. When the ionization chamber and samples were completely surrounded with a shield of cadmium and boron carbide $\left(\mathrm{B}_{4} \mathrm{C}\right)$ the fission rates dropped to negligibly small values.

Worth appending here is the information which we have obtained about the alpha-activity of $94^{239}$. During the decay of the 125-millicurie sample of $93^{239}$ the sample was placed near an ionization chamber connected to a linear, pulse amplifier in order to watch for the growth of alpha-particles. A strong magnetic field was used to bend out the beta-particles. An alpha-particle activity was observed to grow with a half-life of about 2.3 days, which is the half-life to be expected for growth from $93^{239}$. The alpha-count grew to the value 240 per minute. After the sample was thinned to $0.16 \mathrm{mg}$ per $\mathrm{cm}^{2}$ the alphacount became 800 per minute. Correcting for the geometrical factor, as determined with the aid of a known amount of uranium of the same thickness as this sample, the total alpha-emission of the sample amounted to about 60,000 per minute. This corresponds to a half-life of about $3 \times 10^{4}$ years. 